\title{
Periconceptional folic acid supplementation and risk of parent-reported asthma in children at 4-6 years of age
}

\author{
Jufen Liu (1) ${ }^{1,2}$, Zhiwen $\mathrm{Li}^{1,2}$, Rongwei Ye $\mathrm{e}^{1,2}$, Jianmeng Liu ${ }^{1,2}$ and Aiguo Ren (1) ${ }^{1,2}$
}

Affiliations: ${ }^{1}$ Institute of Reproductive and Child Health/Key Laboratory of Reproductive Health, National Health Commission of the People's Republic of China, Peking University, Beijing, PR China. ${ }^{2}$ Dept of Epidemiology and Biostatistics, School of Public Health, Peking University, Beijing, PR China.

Correspondence: Aiguo Ren, 38 College Rd, Haidian District, Institute of Reproductive and Child Health, Peking University Health Science Center, Beijing 100191, China. E-mail: renagabjmu.edu.cn

\section{ABSTRACT}

Background: Folic acid supplementation is universally recommended for women of child-bearing age to prevent fetal neural tube defects (NTDs). Concerns have arisen over the potential risk for childhood allergy and asthma due to folic acid supplementation. We examined whether periconceptional supplementation with low-dose folic acid only was associated with an increased risk for allergy symptoms or asthma in offspring at 4-6 years of age.

Methods: Out of 247831 participating women enrolled in 1993-1996, 9090 were randomly selected and their children were followed up in 2000-2001. Information on mothers' demographic characteristics, folic acid supplementation and allergic diseases among children was collected. We used logistic regression to evaluate the association between folic acid intake and risk for allergic disease while adjusting for potential confounding factors.

Results: The rate of allergy symptoms was $1.54 \%$ among children whose mothers had taken folic acid compared with $2.04 \%$ among those whose mothers had not taken folic acid, and the rate of asthma was $0.92 \%$ and $0.88 \%$, respectively. Maternal folic acid supplementation was not associated with risk for allergy symptoms or asthma, with odds ratios $(95 \%$ CI) of $0.80(0.58-1.11)$ and $1.04(0.67-1.61)$, respectively. No differences in the occurrence of allergy symptoms or asthma were observed when data were analysed by timing of supplementation or compliance with folic acid supplementation.

Conclusions: Supplementation with low-dose folic acid only during the periconceptional period did not increase risk for allergy symptoms or asthma in children at 4-6 years of age in a population without staple fortification with folic acid.

@ERSpublications

A large cohort showed no association between maternal folic acid supplementation and risk of asthma in children http://bit.ly/2RC7cDa

Cite this article as: Liu J, Li Z, Ye R, et al. Periconceptional folic acid supplementation and risk of parent-reported asthma in children at 4-6 years of age. ERJ Open Res 2020; 6: 00250-2019 [https:// doi.org/10.1183/23120541.00250-2019].

This article has supplementary material available from openres.ersjournals.com

Received: 14 Sept 2019 | Accepted after revision: 19 Jan 2020

Copyright $\odot$ ERS 2020. This article is open access and distributed under the terms of the Creative Commons Attribution Non-Commercial Licence 4.0. 


\section{Introduction}

Periconceptional folic acid supplementation has well-established beneficial effects for preventing neural tube defects (NTDs) [1-3]; therefore, it is universally recommended for women of reproductive age, including women in the early stages of pregnancy and those planning to become pregnant. In addition, the strong evidence demonstrating a reduced risk for NTDs as a result of folic acid supplementation has led to mandatory FA fortification of staples in more than 80 countries, including the USA [4], Canada [5] and Chile [6], among others. These interventions have resulted in an unprecedented number of women being exposed to nondietary sources of folic acid $[6,7]$, and concerns have arisen over the potential risk of excess folic acid for childhood allergic disorders [8-11]. Data have shown that the rate of childhood asthma has increased significantly, doubling from 1980 to 1995 and then increasing slowly from 2001 to 2010 in the USA [12]. The same trend has been observed in lower middle-income countries [13], which makes it a critical public health problem.

The findings of studies on a possible association between maternal folic acid supplementation and allergic disorders in children are inconsistent. In a population-based cohort study in Norway, maternal supplementation with folic acid was associated with a slightly increased risk for asthma in children [10]; however, this increased risk was seen only in women who ate a diet rich in folate. A case-control study suggested that maternal intake of folic acid at a dose higher than the recommended dose $\left(>400 \mu \mathrm{g} \cdot \mathrm{day}^{-1}\right)$ was more often observed in the group of allergic subjects than in the control group [14], whereas supplementation with a relatively low dose was associated with a decreased risk for infant asthma (<36 $000 \mu \mathrm{g} \cdot$ day $\approx 400 \mu \mathrm{g} \cdot \mathrm{day}^{-1}$ for less than 3 months, OR $0.36,95 \%$ CI $0.17-0.77$ ) [15]. Finally, in other studies, prenatal folic acid exposure was associated with a higher risk for childhood asthma in countries that did not fortify the food supply with folic acid; the association was not observed in countries where mandatory food fortification with folic acid already existed $[9,16]$.

Differences in the timing of folic acid supplementation and in children's age when allergic disorders were identified have led to variable results. Supplementation in the first trimester of pregnancy slightly increased the risk of wheezing in children younger than 18 months in Norway [17], whereas studies in the USA did not find an association between second-trimester maternal plasma folate levels and infant bronchiolitis [18] or asthma [16]. Folic acid supplementation in late pregnancy (30-34 weeks) was related to an increased risk for asthma at 3.5 years persistent to 5.5 years of age in Australia [19]. Finally, a recent study in the Netherlands showed that maternal use of folic acid during pregnancy was associated an increased risk of wheezing only at 1 year of age; it was not associated with childhood respiratory health or atopy at 8 years old [20].

The discrepancies in these findings may be due to differences in study design (cross-sectional versus longitudinal), sample size, timing of supplementation, dose, child age, or form of folic acid (folic acid only or multiple vitamins). In the present study, we examined whether periconceptional supplementation with low-dose folic acid only $\left(400 \mu \mathrm{g} \cdot \mathrm{day}^{-1}\right)$ was associated with an increased risk for allergy symptoms or asthma in children 4-6 years of age in a large mother-child cohort in a setting with no staple fortification with folic acid.

\section{Methods}

\section{Study design and subjects}

This study was based on a community intervention trial conducted in China during the 1990s that evaluated the effectiveness of folic acid supplementation with no additional vitamins on the prevention of NTDs [1]. The original trial included 247831 women who were registered with a pregnancy-monitoring system (PMS) between October 1993 and September 1995; birth outcomes were tracked through December 31, 1996 [21]. All women in the project counties who were preparing for marriage or who became pregnant were registered and advised to take a pill containing $400 \mu \mathrm{g}$ folic acid only every day. All FA supplementation was supplied by the community intervention project. Supplementation started at the time of registration with the PMS and stopped at the end of the first trimester. For those women who were in the second trimester when registered, no folic acid supplement was available for them. Detailed information on folic acid supplementation was prospectively recorded through checking the number of pills taken at monthly home visits by local health workers, and demographic information about the subjects was collected at registration. From December 2000 to September 2001, when the children born to these women were 4-6 years of age, a total of 9100 mother-child pairs were randomly selected from the PMS [22, 23]. That is, 350 pairs in each city/county from 26 cities/counties that participated in the trial were randomly selected (Supplementary Figure 1). Parents were interviewed in person and asked to complete the Child Behavior Checklist (CBCL), a standardised questionnaire that uses parent ratings to assess emotional and behavioural problems in children and adolescents [24]. All interviewers were trained and passed qualifying examinations before participating in the investigation. We excluded cases with 
missing information on child sex, maternal age, maternal education, or allergic disorders. A total of 9090 mother-child pairs were ultimately included in the final analyses.

\section{Definition of folic acid use}

The classification and patterns of folic acid consumption were the same as previously reported [1]. Briefly, women who had taken folic acid pills at any time from the start of the registration period until the end of the first trimester of pregnancy were classified as folic acid users. Women who had not agreed to take folic acid or who had been registered during the second trimester of pregnancy did not receive folic acid supplements (i.e., did not have the opportunity to start taking folic acid by the end of the first trimester) were considered nonusers. Three patterns of folic acid exposure were differentiated: periconceptional, or initiation before the last menstrual period and cessation within the first trimester; preconceptional, or initiation and cessation before the last menstrual period; and postconceptional, or initiation after the last menstrual period and cessation within the first trimester. Compliance was calculated as the actual number of pills consumed divided by the expected number of pills. Women who had taken more than $80 \%$ of the assigned pills made up the compliant subgroup, and those who had taken less than $80 \%$ of the assigned pills made up the noncompliant subgroup.

\section{Definition of allergic disorders}

Information on allergy symptoms and asthma was obtained from Item 2 (allergy symptoms) and Item 4 (asthma) of the CBCL. The CBCL (Parent version; used for children ages 4-16 years) consists of 113 items with a 3-point Likert scale response format. For each item that describes the child now or within the past 6 months, $0=$ not true, $1=$ somewhat or sometimes true, and $2=$ very true or often true. The items were filled out by parents of children (in most cases, the children's mothers) under the instruction of two interviewers who were trained neurological paediatricians at local maternal and children's hospitals [23, 25]. We defined children as having allergy symptoms (asthma) when Item 2 (Item 4) was rated 2. Another questionnaire collected information on the health and development of the child. Health was measured with a self-report question about hospitalisation and reasons for hospitalisation in the past year. We also included hospitalisation due to asthma as an outcome in the current study.

The original trial and follow-up survey were approved by the institutional review boards of Peking University Health Science Center and the US Centers for Disease Control and Prevention. All women who participated in the original trial and mothers of the children in the follow-up survey provided oral informed consent.

\section{Statistical analyses}

We calculated data on rates of allergy symptoms, asthma, and hospitalisation due to asthma according to patterns of maternal folic acid intake. We estimated odd ratios by dividing the rate of main outcomes among women who had taken folic acid by that among women who had not taken folic acid. Power analyses were conducted to anticipate the likelihood that the study would yield a significant effect. Logistic regression was used to adjust for the main potential confounding variables, including maternal age at child birth, education, occupation and parity. Because rates of childhood allergy symptoms and asthma differ between northern and southern China, we performed further analyses stratified by region. All data were analysed with SPSS (version 24.0; IBM, Chicago, IL, USA).

\section{Results}

Table 1 shows demographic characteristics for the 9090 mothers according to patterns of periconceptional folic acid use. Half of the mothers had taken folic acid during the periconceptional period. Women who had taken folic acid were, on average, 1-2 years younger than those who had not. Women who had taken folic acid supplements were more likely to be farmers and factory workers and to have a junior high school education or higher. There were no significant differences in the proportions of male and female infants between the two groups.

The rate of allergy symptoms was $1.54 \%$ among children whose mothers had taken folic acid compared with $2.04 \%$ among those whose mothers had not taken folic acid $(\mathrm{p}=0.070)$, and the rate of asthma was $0.92 \%$ and $0.88 \%$, respectively. In addition, there was no difference in hospitalisation due to asthma between the two groups (table 2). Power analyses showed that our study size had $93.6 \%$ power $(\alpha=0.15)$ to detect a decrease of $10 \%$ over the unexposed rate of $0.92 \%$ for asthma.

When the data were stratified by the timing of supplementation and compliance with supplementation, there were no significant differences between groups (table 3). The rate of allergy symptoms was $1.19 \%$, $2.18 \%$, and $1.63 \%$ in groups with folic acid supplementation in the periconceptional, preconceptional and 


\begin{tabular}{|c|c|c|c|}
\hline Characteristics & Folic acid users & Non-users & p-value \\
\hline Subjects & 4542 & 4548 & \\
\hline Age at child birth years & $24.8 \pm 2.5$ & $25.9 \pm 3.5$ & $<0.001$ \\
\hline Only child & 4104 (90.5) & $3545(78.0)$ & $<0.001$ \\
\hline \multicolumn{4}{|l|}{ Education } \\
\hline Senior high school or more & 1025 (24.5) & $1214(28.6)$ & $<0.001$ \\
\hline Junior high school & $2325(55.6)$ & $2012(47.4)$ & \\
\hline Primary school or less & $829(19.8)$ & $1022(24.1)$ & \\
\hline \multicolumn{4}{|l|}{ Occupation } \\
\hline Farmer & 1579 (34.8) & 1585 (34.9) & 0.001 \\
\hline Factory worker & $1771(39.0)$ & 1615 (35.5) & \\
\hline Other or unknown & $1192(26.2)$ & $1348(29.6)$ & \\
\hline \multicolumn{4}{|l|}{ Sex of infant } \\
\hline Male & $2382(52.4)$ & $2390(52.6)$ & 0.919 \\
\hline Female & $2160(47.6)$ & $2158(47.4)$ & \\
\hline
\end{tabular}

postconceptional periods, respectively. This rate was $1.43 \%$ in the compliant group (compliance $\geqslant 80 \%$ ) compared $0.88 \%$ in the noncompliant group (compliance $<80 \%$ ).

When age, number of children, education, and occupation were adjusted for, folic acid supplementation was not associated with risk for allergy symptoms, asthma or hospitalisation due to asthma in the current population, with OR $(95 \% \mathrm{CI})$ of $0.80(0.58-1.11), 1.04(0.67-1.61)$, and $0.79(0.35-1.77)$, respectively (table 4).

TABLE 2 Rate of allergic symptoms, asthma and hospitalisation due to asthma among offspring by maternal folic acid supplementation, China, 2000-2001

\begin{tabular}{|c|c|c|c|c|}
\hline & \multicolumn{2}{|c|}{ Folic acid } & \multirow[t]{2}{*}{ p-value } & \multirow[t]{2}{*}{ Total } \\
\hline & Yes & No & & \\
\hline Subjects & 4542 & 4548 & & 9090 \\
\hline Allergic symptoms & $70(1.54)$ & $93(2.04)$ & 0.070 & $163(1.79)$ \\
\hline Asthma & $42(0.92)$ & $40(0.88)$ & 0.820 & $82(0.90)$ \\
\hline Hospitalisation due to asthma & $11(0.24)$ & $16(0.35)$ & 0.337 & $27(0.30)$ \\
\hline
\end{tabular}

TABLE 3 Compliance of maternal folic acid supplementation and risk for allergic symptoms, asthma and hospitalisation due to asthma in offspring, 2000-2001 n (\%)

\section{Folic acid}

\section{Periconception Preconception}

Subjects

Allergic symptoms

Asthma

Hospitalisation due to asthma

\begin{tabular}{cccc}
\hline & Preconception & Postconception & $\begin{array}{c}\mathbf{p} \\
\text { Value }\end{array}$ \\
\hline 2360 & 229 & 1101 & \\
$28(1.19)$ & $5(2.18)$ & $18(1.63)$ & 0.384 \\
$15(0.64)$ & $1(0.44)$ & $10(0.91)$ & 0.765 \\
$4(0.17)$ & $2(0.87)$ & $10(0.91)$ & 0.479
\end{tabular}

Compliance $^{\#}$

$\begin{array}{ccc}\begin{array}{c}\text { Not compliant } \\ \text { (340) }\end{array} & \begin{array}{c}\text { Compliant } \\ \text { (3354) }\end{array} & \begin{array}{c}\mathbf{p} \\ \text { Value }\end{array} \\ 340 & 3354 & \\ 3(0.88) & 48(1.43) & 0.443 \\ 4(1.18) & 22(0.66) & 0.522 \\ 0(0) & 4(0.12) & 0.565\end{array}$

Data are presented as $\mathrm{n}$ or $\mathrm{n}(\%)$, unless otherwise stated. Values for some characteristics may not be equal to total numbers in each group because of missing values. ": Compliance was calculated as actual consumed pills divided by expected pills, compliant refers to compliance $>80 \%$ and not compliant refers to compliance $<80 \%$. 
TABLE 4 Odds ratio of allergic symptoms, asthma and hospitalisation due to asthma among offspring by maternal folic acid supplementation, China, 2000-2001

\begin{tabular}{lcc} 
Disease & Unadjusted odds ratio $(95 \% \mathrm{Cl})$ & ${\text { Adjusted odds ratio }\left(95 \% \mathrm{CI}^{\#}\right.}^{*}$ \\
\hline Allergic symptoms & $0.75(0.55-1.03)$ & $0.80(0.58-1.11)$ \\
Asthma & $1.05(0.68-1.63)$ & $1.04(0.67-1.61)$ \\
Hospitalisation due to asthma & $0.69(0.32-1.48)$ & $0.79(0.35-1.77)$ \\
\hline \#: Adjusted by age, only-child, education and occupation. & \\
\hline
\end{tabular}

Because the rate of allergy symptoms was higher in southern China (2\%) than northern China (0.7\%), we repeated the analyses stratified by region. The results did not differ from the overall analyses (Supplementary Tables).

\section{Discussion}

Although beneficial effects of folic acid for preventing NTDs are well documented [1, 2, 26], concerns have arisen over increased risk for childhood allergic disorders due to maternal folic acid supplementation. Our study was conducted in a population with low blood folate concentrations: red blood cell folate concentrations were $440.0 \mathrm{nmol} \cdot \mathrm{L}^{-1}$ in the north and $910.4 \mathrm{nmol} \cdot \mathrm{L}^{-1}$ in the south in 2003 [27]. Among the 9090 mother-child pairs, we found that a low dose of periconceptional folic acid supplementation ( $400 \mu \mathrm{g}$ FA only) every day did not increase risk for allergy symptoms or asthma in overall analyses or when the timing of supplementation or compliance was taken into consideration.

Of the possible unintended effects of folic acid supplementation, allergic disorders and asthma are noteworthy. Findings in mouse models have motivated more than 20 population studies on folate and asthma [8, 28]; however, these studies have differed in terms of design, sample size, measurement of exposure and outcome (questionnaire assessment or physical examination), timing or dosage of supplementation, and other confounding factors, which makes the results inconclusive. A recent large, prospective population-based cohort study showed that maternal supplementation with folic acid was associated with a slightly increased risk for asthma in children; however, the study was conducted in a population with high folate levels [10]. A meta-analysis of 16 cohort, seven case-control and three cross-sectional studies revealed that maternal folic acid supplementation was not associated with the development of asthma among offspring, whereas exposure during early pregnancy was related to wheezing occurrence in offspring (relative risk=1.06, 95\% CI 1.02-1.09) [29]. A recent meta-analysis of reports in eight electronic databases that examined the association between prenatal folate or folic acid exposure and risk for asthma and allergy in childhood provided no evidence of an association between maternal folic acid supplementation (compared with no use of folic acid) in the prepregnancy period through the first trimester and asthma in childhood (summary risk estimate: 1.01, 95\% CI 0.78-1.30) [28], in line with our results. Although there are inadequate data to exclude a weak effect of maternal folate on asthma, such an effect should be examined within the context of very large and ongoing birth cohort studies. Our study provides additional evidence from a large Asian cohort, which enriches the literature.

One unique characteristic of the present study is that the supplement contained a low dose of folic acid only $(400 \mu \mathrm{g}$ per pill). First, this enabled us to exclude the possibility of effects of other vitamins or minerals contained in the micronutrients used in most other published studies. Besides the possible effect of folic acid supplementation in pregnant women on childhood allergic disease, studies have shown that nutrition has both direct and indirect effects on sensitisation to allergy [30]. A meta-analysis showed that increasing maternal vitamin D intake during pregnancy had a protective effect on wheezing and asthma in children [31]. Although vitamin E is not consistently associated with asthma and/or allergy [32], polyunsaturated fatty acids and prebiotics may protect against the development of allergy [33]. Secondly, we found that a low dose of folic acid supplementation did not increase allergic disorder in offspring. In contrast, previous studies have revealed associations mainly among women using a higher dose of folic acid than recommended $\left(>400 \mu \mathrm{g} \cdot \mathrm{day}^{-1}\right)[14,15]$ or among pregnant women taking supplemental folic acid above the recommended dose in combination with a diet rich in folate [10].

Another characteristic of this study is that the population was not exposed to staples fortified with folic acid or other micronutrients, as in many other published reports. In addition to the possibility that other micronutrients in fortified staples cause allergy, a higher intake of folic acid from fortified staples may be a concern [14]. Our study was conducted when there was no mass folic acid supplementation or food fortification among women of child-bearing age in China, and few women consumed multiple vitamins at 
the time [34]; the only intervention was supplementation with folic acid only ( $400 \mu \mathrm{g}$ folic acid per tablet). Hence, our study provides evidence that recommended folic acid supplementation among mothers did not increase allergy symptoms or asthma among offspring in a large population with low folate levels.

Although there was a significant difference in the rate of allergy symptoms between northern and southern China $(0.8 \%$ versus $2.8 \%)$, the overall rate in China is low compared to the global prevalence $(11.7 \%)$ among children 6-7 years old [35]. The aetiology of allergy symptoms and asthma remains largely unknown and is generally thought to be affected by multiple factors. Food/diet, constitution, environment and other factors contribute to the condition. Animal studies have revealed that elevated methyl donor availability (i.e. supplementation with folic acid, vitamin B12, choline, L-methionine, zinc and betaine in late pregnancy) increases susceptibility to allergy in progeny [36]. Studies are needed to determine whether folic acid supplementation in pregnant women is associated with patterns of DNA methylation in their children similar to those found in mouse pups and whether these DNA changes are also associated with increased childhood asthma [37]. Moreover, because the doses of folic acid supplementation vary by in different settings varies, future studies must verify whether the asthma to be correlated with high and/or low folate levels needs to verify in the future study.

The present study has several strengths. First, it was based on a well-organised, population-based PMS that used quality-control measures to ensure data quality [1]. Information on folic acid supplementation was reliably recorded by local healthcare workers. The enrolled women were advised to take a pill containing $400 \mu \mathrm{g}$ folic acid every day, starting at the time of registration with the PMS and continuing until they completed the first trimester of pregnancy. If women consented to take folic acid, the pills were distributed at the time of registration. At the end of each month, local health workers recorded the dates of all menstrual periods and how many pills remained in each bottle (if women were taking pills). Secondly, folic acid was the only intervention and the supplementation was till to first trimester; no other vitamins or minerals were investigated. Thus, the results reflect the pure and sole effect of folic acid only. There were no over-the-counter folic acid supplements in China at that time; all folic acid supplementation was supplied by the community intervention project. For all the folic acid users, the supplementation lasted till the end of first trimester, after which, no folic acid supplements would be provided. For those women who were in the second trimester when registered, no folic acid supplement was available. Hence, there was no one who consumed folic acid supplements during their second trimester in our study. So, we can only observe the potential effect of folic acid supplementation in the first trimester. The dose of folic acid used in our study was $400 \mu \mathrm{g}$. We were able to identify effects associated with this dose, which is a widely recommended dose. During the intervention, no other vitamins were available, which precludes the possibility of confounding by multiple vitamins. Thirdly, in the follow-up study, mother-child pairs were randomly selected. Interviewers were systematically trained, which helped reduce inconsistencies in outcome assessment.

However, a couple of limitations should also be noted. First, we did not collect information on the dietary folate intake of the women, which could have influenced the results. However, the study population had low folate levels, and there was no other folic acid fortification besides the current intervention. Secondly, confounding by factors associated with folic acid use remains a possibility. We did not collect information on factors such as maternal smoking or maternal asthma. However, the prevalence of smoking and asthma among Chinese women is quite low, only 3.4\% for smoking in 2010 [38] and 2.6\% for asthma in 2015 [38, 39], and adjusting for several demographic factors did not change our results appreciably. Thirdly, the CBCL is not a diagnostic tool for allergy or asthma; in preschool children, correct diagnosis of such disorders remains difficult. However, the use of CBCL effectively standardises parent reports, and is likely the most appropriate practical means in a study of this nature. Therefore, the overall results are unlikely to be impacted by the use of CBCL because both intervention and control groups were assessed in the same way. While related, asthma and allergy are different conditions, and distinguishing between allergic and nonallergic asthma in 4-6 year olds can be highly complex. As results from previous studies have revealed that folic acid supplementation is not associated with asthma but is associated with wheezing [29], the definition and measurement of allergy and asthma in different studies vary and need to receive more attention in further analyses. The development of a reliable and convenient screening method that can be used in large investigations should be a subject of future study.

In conclusion, in this large follow-up study, we did not observe an increase in risk for allergy symptoms or asthma among offspring born to women who had taken a recommended low dose of folic acid during the periconceptional period in a population without folic acid fortification, thus providing evidence of the safety of supplementation with low-dose folic acid for preventing NTDs. In fact, the Ministry of Health of China initiated a nationwide folic acid supplementation programme in 2009 [40]. Women who plan to become pregnant are eligible to obtain folic acid supplements for 6 months free of charge through the maternal healthcare system $[41,42]$. It is estimated that more than 100 million women of reproductive age 
participated in the programme from 2009 to 2018 [43], resulting in a marked increase in blood folate [44, 45] and a substantial decrease in the prevalence of NTDs in high-prevalence areas [42]. Currently no staples are fortified with folic acid in China, but this should be an alternative strategy for maximising prevention of NTDs, which are prevalent in northern parts of the country.

Author contributions: J. Liu analysed the data, drafted the initial manuscript and revised the manuscript. A. Ren conceptualised the study and critically revised the manuscript. $\mathrm{Z}$. Li contributed to data analysis and reviewed the manuscript. A. Ren, R. Ye and J. Liu coordinated and supervised data collection. All authors approved the final manuscript as submitted and agree to be accountable for all aspects of the work.

\section{Conflict of interest: None declared.}

Support statement: This work was supported in part by the National Key Research and Development Program, Ministry of Science and Technology of the People's Republic of China (grant number 2016YFC1000501) and Natural Science Foundation of China (number 81202265). The original project was supported by a cooperative agreement between the US Centers for Disease Control and Prevention and Peking University (number U01 DD000293). Funding information for this article has been deposited with the Crossref Funder Registry.

\section{References}

1 Berry RJ, Li Z, Erickson JD, et al. Prevention of neural-tube defects with folic acid in China. China-U.S. Collaborative Project for Neural Tube Defect Prevention. N Engl J Med 1999; 341: 1485-1490.

2 Czeizel AE, Dudas I. Prevention of the first occurrence of neural-tube defects by periconceptional vitamin supplementation. N Engl J Med 1992; 327: 1832-1835.

3 Brinkhof M, Meier S, Chamberlain J, et al. The impact of folic acid food fortification on global incidence of neural tube defects: systematic review, meta-analysis and estimate of preventable cases. Int J Epidemiol 2015; 44: 143.

$4 \quad$ Wald NJ. Folic acid and the prevention of neural-tube defects. N Engl J Med 2004; 350: 101-103.

5 Plumptre L, Masih SP, Ly A, et al. High concentrations of folate and unmetabolized folic acid in a cohort of pregnant Canadian women and umbilical cord blood. Am J Clin Nutr 2015; 102: 848-857.

6 Martorell R, de Romana DL. Components of successful staple food fortification programs: lessons from Latin America. Food Nutr Bull 2017; 38: 384-404.

7 Flores AL, Cordero AM, Dunn M, et al. Adding folic acid to corn masa flour: Partnering to improve pregnancy outcomes and reduce health disparities. Prev Med 2018; 106: 26-30.

8 Blatter J, Han YY, Forno E, et al. Folate and asthma. Am J Respir Crit Care Med 2013; 188: 12-17.

9 Han YY, Celedon JC. Maternal folate intake during pregnancy and childhood asthma. Am J Respir Crit Care Med 2017; 195: 155-156.

10 Parr CL, Magnus MC, Karlstad O, et al. Maternal folate intake during pregnancy and childhood asthma in a population-based cohort. Am J Respir Crit Care Med 2017; 195: 221-228.

11 McStay CL, Prescott SL, Bower C, et al. Maternal folic acid supplementation during pregnancy and childhood allergic disease outcomes: a question of timing? Nutrients 2017; 9: 123.

12 Akinbami LJ, Simon AE, Rossen LM. Changing trends in asthma prevalence among children. Pediatrics 2016; 137: e20152354.

13 Fischer GB, Sarria EE, Camargos P, et al. Childhood asthma in low and middle-income countries: where are we now? Paediatr Respir Rev 2019; 31: 52-57.

14 Socha-Banasiak A, Kamer B, Paczes K, et al. Trends in folic acid supplementation during pregnancy - the effect on allergy development in children. Postepy Dermatol Alergol 2018; 35: 139-144.

15 Yang L, Jiang L, Bi M, et al. High dose of maternal folic acid supplementation is associated to infant asthma. Food Chem Toxicol 2015; 75: 88-93.

16 Trivedi MK, Sharma S, Rifas-Shiman SL, et al. Folic acid in pregnancy and childhood asthma: a US cohort. Clin Pediatr (Phila) 2018; 57: 421-427.

17 Haberg SE, London SJ, Stigum H, et al. Folic acid supplements in pregnancy and early childhood respiratory health. Arch Dis Child 2009; 94: 180-184.

18 Vereen S, Gebretsadik T, Johnson N, et al. Association between maternal 2nd trimester plasma folate levels and infant bronchiolitis. Matern Child Health J 2019; 23: 164-172.

19 Whitrow MJ, Moore VM, Rumbold AR, et al. Effect of supplemental folic acid in pregnancy on childhood asthma: a prospective birth cohort study. Am J Epidemiol 2009; 170: 1486-1493.

20 Bekkers MB, Elstgeest LE, Scholtens S, et al. Maternal use of folic acid supplements during pregnancy, and childhood respiratory health and atopy. Eur Respir J 2012; 39: 1468-1474.

21 Li S, Moore CA, Li Z, et al. A population-based birth defects surveillance system in the People's Republic of China. Paediatr Perinat Epidemiol 2003; 17: 287-293.

22 Zhang Y, Jin L, Liu JM, et al. Maternal haemoglobin concentrations before and during pregnancy as determinants of the concentrations of children at 3-5 years of age: A large follow-up study. Eur J Clin Nutr 2018; 73: $1102-1109$.

23 Huang C, Martorell R, Ren A, et al. Cognition and behavioural development in early childhood: the role of birth weight and postnatal growth. Int J Epidemiol 2013; 42: 160-171.

24 Achenbach TM. Child Behavior Checklist 4-18. Burlington, ON, University of Vermont, 1991.

25 Li HT, Ye R, Achenbach TM, et al. Caesarean delivery on maternal request and childhood psychopathology: a retrospective cohort study in China. BJOG 2011; 118: 42-48.

26 Medical Research Council Vitamin Study Research Group. Prevention of neural tube defects: results of the Medical Research Council Vitamin Study. MRC Vitamin Study Research Group. Lancet 1991; 338: 131-137.

27 Ren A, Zhang L, Hao L, et al. Comparison of blood folate levels among pregnant Chinese women in areas with high and low prevalence of neural tube defects. Public Health Nutr 2007; 10: 762-768. 
Crider KS, Cordero AM, Qi YP, et al. Prenatal folic acid and risk of asthma in children: a systematic review and meta-analysis. Am J Clin Nutr 2013; 98: 1272-1281.

29 Wang T, Zhang HP, Zhang X, et al. Is folate status a risk factor for asthma or other allergic diseases? Allergy Asthma Immunol Res 2015; 7: 538-546.

30 Neerven R, Savelkoul H. Nutrition and allergic diseases. Nutrients 2017; 9: 762.

31 Shi D, Wang D, Meng Y, et al. Maternal vitamin D intake during pregnancy and risk of asthma and wheeze in children: a systematic review and meta-analysis of observational studies. J Matern Fetal Neonatal Med 2019; in press [https://doi.org/10.1080/14767058.2019.1611771].

32 Caraffa AL, Varvara G, Spinas E, et al. Is vitamin E an anti-allergic compound? J Biol Regul Homeost Agents 2016; 30: $11-15$.

33 Thorburn AN, McKenzie CI, Shen S, et al. Evidence that asthma is a developmental origin disease influenced by maternal diet and bacterial metabolites. Nat Commun 2015; 6: 7320.

34 Li Z, Ye R, Zhang L, et al. Periconceptional folic acid supplementation and the risk of preterm births in China: a large prospective cohort study. Int J Epidemiol 2014; 43: 1132-1139.

35 Mallol J, Crane J, von Mutius E, et al. The International Study of Asthma and Allergies in Childhood (ISAAC) phase three: a global synthesis. Allergol Immunopathol (Madr) 2013; 41: 73-85.

36 Gatford KL, Wooldridge AL, Kind KL, et al. Pre-birth origins of allergy and asthma. J Reprod Immunol 2017; 123: 88-93.

37 Ownby DR. Has mandatory folic acid supplementation of foods increased the risk of asthma and allergic disease? J Allergy Clin Immunol 2009; 123: 1260-1261.

38 Liu S, Zhang M, Yang L, et al. Prevalence and patterns of tobacco smoking among Chinese adult men and women: findings of the 2010 national smoking survey. J Epidemiol Community Health 2017; 71: 154-161.

39 Loerbroks A, Ding H, Han W, et al. Work stress, family stress and asthma: a cross-sectional study among women in China. Int Arch Occup Environ Health 2017; 90: 349-356.

40 Ministry of Health China. 2012. China Birth Defects Prevention report (2012). [In Chinese].

41 Ren AG. Prevention of neural tube defects with folic acid: The Chinese experience. World J Clin Pediatr 2015; 4: 41-44.

42 Liu J, Zhang L, Li Z, et al. Prevalence and trend of neural tube defects in five counties in Shanxi province of Northern China, 2000 to 2014. Birth Defects Res Part A Clin Mol Teratol 2016; 106: 267-274.

43 National Health Commission. 2019. Report on the development of maternal and child health in China (2019) [in Chinese].

44 Liu JF, Gao LL, Zhang YL, et al. Plasma folate levels in early to mid pregnancy after a nation-wide folic acid supplementation program in areas with high and low prevalence of neural tube defects in china. Birth Defects Res Part A Clin Mol Teratol 2015; 103: 501-508.

45 Zhang XJ, Liu JF, Jin YS, et al. Folate of pregnant women after a nationwide folic acid supplementation in China. Mater Child Nutr 2019; 15: e12828. 